\title{
The role of $\mathrm{PET} / \mathrm{CT}$ imaging in penile cancer
}

\author{
Sarah R. Ottenhof ${ }^{1}$, Erik Vegt ${ }^{2}$ \\ ${ }^{1}$ Department of Urology, Netherlands Cancer Institute, Amsterdam, the Netherlands; ${ }^{2}$ Department of Nuclear Medicine, Netherlands Cancer \\ Institute, Amsterdam, the Netherlands \\ Contributions: (I) Conception and design: All authors; (II) Administrative support: None; (III) Provision of study materials or patients: All authors; \\ (IV) Collection and assembly of data: SR Ottenhof; (V) Data analysis and interpretation: All authors; (VI) Manuscript writing: All authors; (VII) Final \\ approval of manuscript: All authors. \\ Correspondence to: Erik Vegt. Department of Nuclear Medicine, Netherlands Cancer Institute, Amsterdam, the Netherlands. Email: e.vegt@nki.nl.
}

\begin{abstract}
Positron emission tomography (PET) imaging with ${ }^{18} \mathrm{~F}$-fluorodeoxyglucose (FDG) combined with computed tomography (CT) provides functional imaging combined with anatomic information, improving diagnostic accuracy and confidence. Although virtually all primary penile tumors are FDG-avid, PET/CT is not recommended for primary tumor staging as it has limited spatial resolution and is hampered by urinary FDG excretion. The accuracy of PET/CT for lymph node staging seems to improve with the pretest likelihood of metastatic nodes. In groins with normal physical examination, sensitivity is only $57 \%$. In groins with palpably enlarged lymph nodes, sensitivity of PET/CT reaches $96 \%$. For pelvic lymph nodes and distant metastases, PET/CT is more accurate if inguinal metastases are present. However, these results are based on a very limited number of studies. Overall, the role of PET/CT imaging in penile cancer remains ambiguous, especially in inguinal lymph nodes. During staging and follow-up, it may be particularly useful in detecting pelvic lymph node metastases and occult distant metastases prior to systemic chemotherapy and/or extensive surgery, improving selection of patients that are most likely to benefit from such therapies.
\end{abstract}

Keywords: Penile cancer; imaging; PET/CT; fluorodeoxyglucose; ${ }^{18} \mathrm{~F}$-fluorodeoxyglucose (FDG)

Submitted Mar 30, 2017. Accepted for publication Apr 03, 2017.

doi: $10.21037 /$ tau.2017.04.36

View this article at: http://dx.doi.org/10.21037/tau.2017.04.36

\section{Introduction}

Cancer of the penis is a rare disease with an incidence of 0.6-2.1 in Western countries (1-3). The majority of these cancers are squamous cell carcinoma, but other histological types and subtypes such as basaloid and warty squamous cell, and melanoma can occur. The worldwide incidence has significant geographic variations, which is mainly explained by circumcision, sexual practices and socioeconomic circumstances (2).

Imaging and other staging techniques have improved the risk stratification of penile cancer. Ultrasound-guided fine needle aspiration biopsy, (dynamic) sentinel node biopsy, and various forms of lymph node dissections are invasive techniques that have improved lymph node staging. In addition, axial imaging such as CT, MRI and PET/CT have also evolved in the past decades.
Positron emission tomography (PET) imaging with ${ }^{18} \mathrm{~F}$-fluorodeoxyglucose (FDG) is based on cellular uptake of glucose and FDG, which is elevated in malignant cells and other tissues with an elevated glycolytic rate (4). FDGPET combined with computed tomography $(\mathrm{CT})$ provides functional imaging combined with anatomic information, improving diagnostic accuracy and confidence. PET/CT scanners acquire PET and CT images in a single scanner, and are nowadays widely used.

In various malignant diseases, $\mathrm{PET} / \mathrm{CT}$ is used for staging and monitoring. Combining functional with anatomical imaging, PET/CT outperforms both PET alone and $\mathrm{CT}$ alone in lymph node staging of many types of tumors (5-7). Here we review the role of PET/CT in the diagnosis and management of penile cancer.

In penile cancers, both the primary tumors and lymph node metastases generally show high uptake of FDG. 

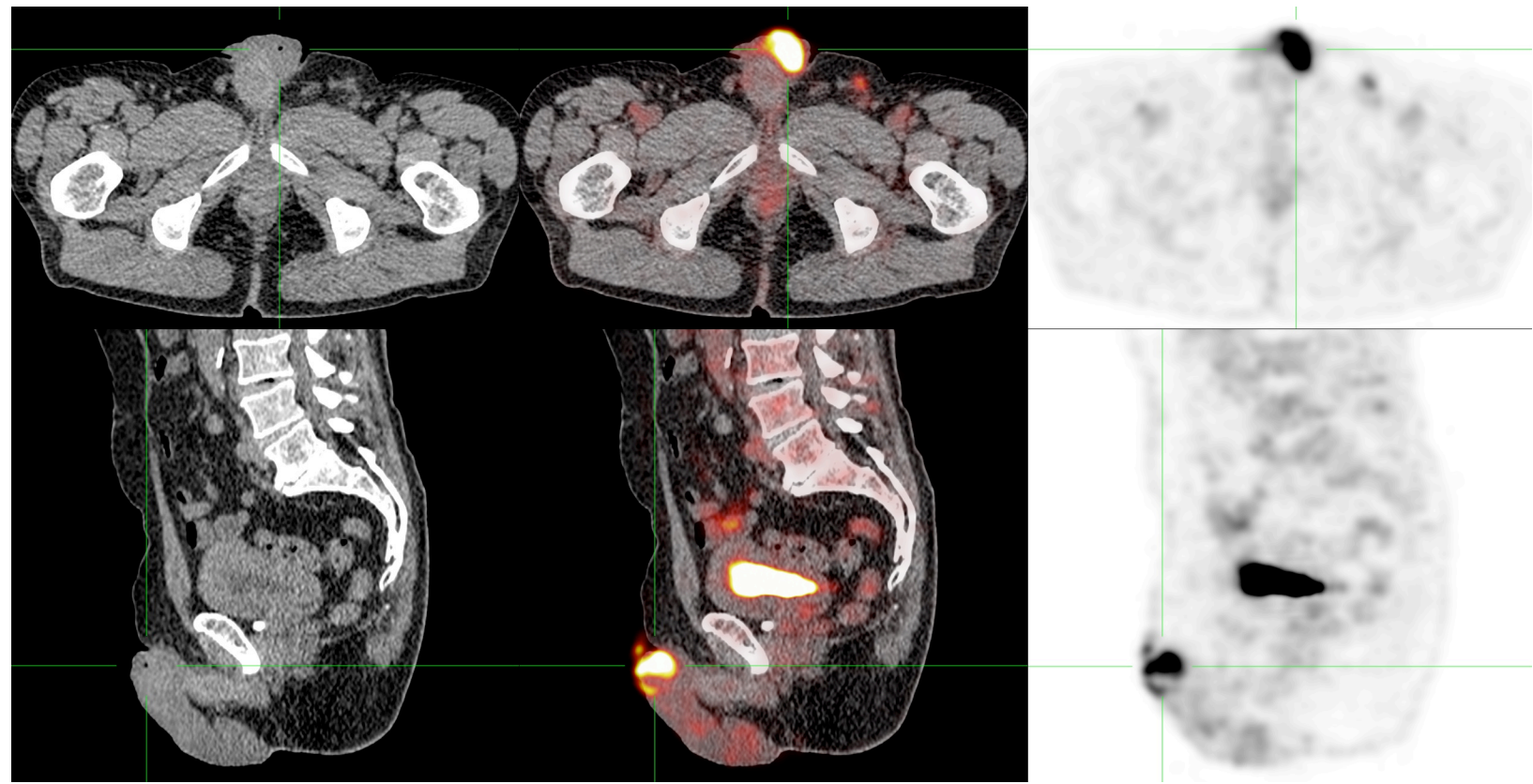

Figure 1 FDG-PET/CT of a penile cancer patient. The primary tumor exhibits high FDG uptake (SUVmax 11.7).

Hence, suitability for staging penile cancer is likely (8). However, sensitivity of PET/CT is limited by its spatial resolution, reducing sensitivity for small metastases. In addition, false-positives may occur due to inflammation, such as in reactive lymph nodes.

\section{Primary tumor staging}

In their first study on PET in penile cancer, Scher et al. found that only 6 out of 8 primary tumors were detected by FDGPET/CT (8), but this can be explained by the small size of the two undetected tumors, which was below the spatial resolution of PET (one residual lesion after incomplete resection of a T1 tumor, and one very small $(<0.5 \mathrm{~cm})$ $\mathrm{T} 1$ tumor. The authors even describe that in retrospect both lesions did show some FDG-uptake.

In our experience, almost all primary penile tumors are FDG-avid. An example is shown in Figure 1. However, PET/CT is not very useful for primary tumor staging, because its limited spatial resolution makes it unsuitable for evaluating ingrowth in surrounding structures or even the exact size of the tumor. Additionally, FDG is excreted via the urine, which can interfere with imaging of the primary tumor. Physical examination and, in selected cases MRI, have excellent accuracy for staging primary penile tumors (9).

\section{Lymph node staging}

The diagnostic value of FDG-PET/CT for lymph node staging varies in different types of cancer. Often, reported sensitivities are higher than for conventional modalities such as CT and MRI, but are not always satisfactory (4). In pancreatic cancer for example, PET/CT shows a sensitivity in N-staging of only $30 \%$ (10). Somewhat better results appear in clinically lymph node negative non-small cell lung cancer (stage I) and head and neck cancer (cN0) patients, with sensitivities for regional lymph nodes of $57 \%$ and $67 \%$ respectively $(11,12)$. Specificities may be higher, but identification of patients where invasive staging or therapy can be omitted requires high sensitivity.

\section{Inguinal lymph nodes}

\section{Non-palpable lymph nodes}

Detection of inguinal involvement using FDG-PET/CT in penile cancer patients that were initially staged as node negative ( $\mathrm{cN} 0$ ) was evaluated in several studies.

In 2005, Scher et al. examined thirteen mostly cN0 patients. Of 16 positive lymph nodes, FDG PET/CT correctly identified 15 as positive, with only one false negative lesion, resulting in a sensitivity per lesion of $94 \%$. 




Figure 2 FDG-PET/CT of the same penile cancer patient, who was clinically staged as positive for inguinal lymph node metastases (cN+). The PET shows extensive pelvic lymph node metastases. Bilateral inguinal metastases were also FDG-positive.

The sensitivity for superficial inguinal lymph nodes was $89 \%$ per lesion and $80 \%$ per patient (8).

Later, Leijte et al. published PET/CT results of solely cN0 groins. Five out of 42 groins had lymph node metastases, but PET/CT only identified one, resulting in a sensitivity of only $20 \%$. The true positive lymph node metastasis was larger than the other four (30 vs. 10, 10, 1 and $1 \mathrm{~mm}$ ). This study emphasizes the limited sensitivity of PET/CT in detecting small metastases (13).

In 2012, Souillac et al. showed better sensitivity for PET/CT in cN0 patients, albeit in a small set of patients. PET/CT correctly identified 3 out of 4 metastases (75\% sensitivity) (14).

In all these three studies, specificity of PET/CT was higher than sensitivity: Scher et al. 100\%, Leijte et al. 92\% and Souillac et al. $88 \%$. Negative predictive values (NPV) were $89 \%, 90 \%$ and $83 \%$ respectively.

In a meta-analysis of seven studies by Sadeghi et al., the pooled sensitivity per groin for FDG-PET/CT in cN0 patients was only $57 \%$ (15). Positive predictive values were also disappointing (between 25 and 37 percent). Therefore, surgical staging is necessary to identify small inguinal lymph node metastases and FDG-PET/CT is not recommended for staging of $\mathrm{cN} 0$ patients $(8,13-15)$.

\section{Palpable lymph nodes}

For patients that present with palpable lymph nodes in the groin, PET/CT has a different clinical value (Figure 2). The aforementioned meta-analysis by Sadeghi et al. found that detection of inguinal metastases with FDG-PET/CT in clinically suspicious groins had a pooled sensitivity and specificity of 96 and 100\% per groin respectively (15). Thus, PET/CT is more accurate in patients with palpable lymph nodes. Assessing the number of lymph nodes involved with PET/CT may be useful when neoadjuvant chemotherapy will be considered in case of multiple or bulky inguinal metastases (16).

\section{Pelvic lymph nodes}

On pelvic lymph nodes, literature is scarce. In a study on 18 patients with proven inguinal metastases, the diagnostic accuracy of FDG-PET/CT was evaluated for staging pelvic lymph nodes (17). The clinical benchmark this was compared to was either histopathology, clinical followup, or radiologic imaging. Twenty-eight of 36 pelvic basins were eligible for analysis, and results were good. Analysis showed a sensitivity of $91 \%$, specificity of $100 \%$, and similar negative and positive predictive values (94 and 100 percent respectively). FDG-PET/CT gave a correct prediction in 


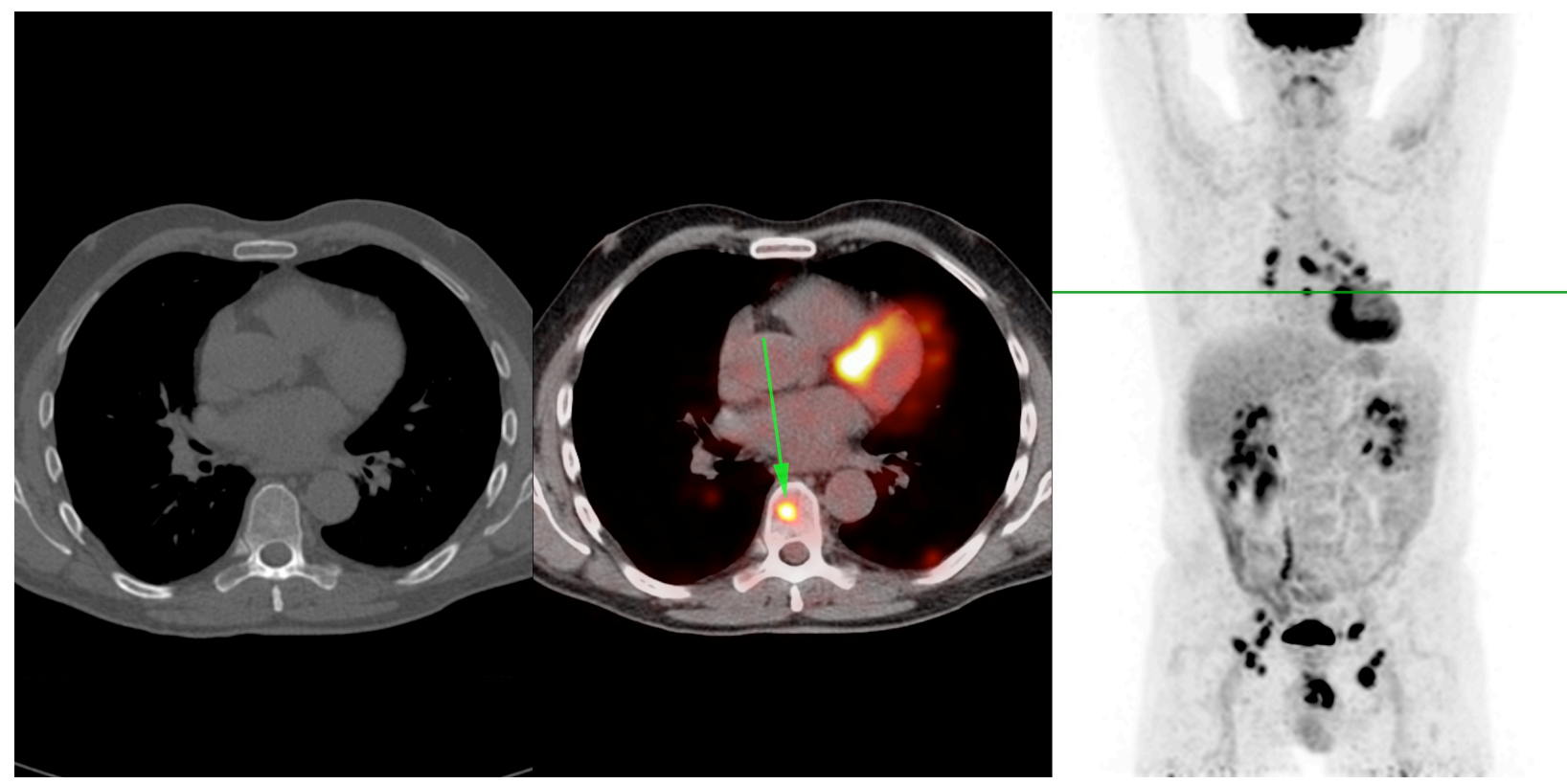

Figure 3 FDG-PET/CT of the same penile cancer patient, showing a solitary skeletal metastasis in the 8th thoracic vertebra, which was not visible on CT. In addition, extensive mediastinal and hilar lymphadenopathy was visible with small pulmonary and pleural lesions, which were thought to be possible sarcoidosis or metastases. Follow-up CT after 3 months showed gross progression, with multiple metastases in bone, liver, spleen and pelvic lymph nodes. In contrast, the lesions in the lungs and mediastinum were stable, increasing the likelihood of those being caused by a separate process such as sarcoidosis.

$96 \%$. This is the only study that provides numbers on pelvic lymph node evaluation of PET/CT.

In stage 3 and 4 patients, Zhang et al. showed good results for lymph nodes (all locations) with a sensitivity of $93 \%$ and a specificity of $85 \%$ (18). Also in other malignancies and lymph node areas, results suggest that FDG-PET/CT has higher accuracy than CT only in staging pelvic lymph nodes $(5,6,19,20)$. However, results are not always optimistic. A prospective multicenter trial in advanced cervical cancer found no significant improvement of sensitivity nor specificity for detection of pelvic lymph node metastases with adding FDG-PET/CT to diagnostic contrast enhanced CT (21). In addition, Lin et al. found lower diagnostic accuracy for PET/CT than CT or MRI in a per scan receiver operating characteristic (ROC) curve analysis for metastatic pelvic lymph nodes or distant metastases in vulvar carcinoma (22).

\section{Distant metastases}

Literature on distant penile cancer metastases detected with FDG-PET/CT consists largely of case reports (23-26).
In the aforementioned study by Graafland et al. among 18 patients with pathologically confirmed inguinal lymph node metastases, five patients were diagnosed with distant metastases by PET/CT (17). Four of these five metastases were confirmed with either CT alone or histopathology (17). Although this was a small number of patients, these results suggest that PET/CT is useful for accurate M-staging.

The largest series by Zhang et al. [2016] compared FDG$\mathrm{PET} / \mathrm{CT}$ to conventional CT in 42 patients with suspicious lesions on CT or MRI, leading to an implicit selection bias (18). With histopathology or follow-up imaging as a reference, sensitivity and specificity of FDG-PET/CT were $85 \%$ and $86 \%$ respectively for all metastatic sites, including lymph nodes, lung, bone, etc. In a patient-based analysis this was $82 \%$ and $93 \%$ respectively. Drawbacks of this study are a short follow-up of patients as well as the aforementioned selection bias.

The whole body imaging aspect of PET/CT scanning can be of crucial impact on clinical decision making. If distant metastases are found in patients scheduled for locoregional therapy (Figure 3), this changes their indicated treatment to systemic or palliative regimens (18). 


\section{Other settings}

\section{Surveillance}

In general, surveillance after treatment of penile cancer depends on physical examination by the physician and the patient himself. In our experience, PET/CT can be of value for identification of the location and extent of suspected recurrence. Routine surveillance with PET/CT is not recommended.

\section{Combined modalities}

A recent study with 129 patients used FDG-PET/CT scanning complementary to sentinel node biopsy (27). In this cohort, FDG-PET/CT found one additional metastatic lymph node that was not detected with the radiotracer of the sentinel node biopsy procedure. The combined procedure had a sensitivity of $94 \%$, reducing the false negative rate to an acceptable level.

\section{Conclusions and future perspectives}

In conclusion, the exact role of FDG-PET/CT imaging in penile cancer remains ambiguous. So far, PET/CT has shown to have significant limitations, especially in inguinal lymph node staging. Results seem to improve with the pre-test likelihood of metastatic involvement.

PET/CT may be particularly useful in assessing pelvic metastases and occult distant metastatic burden prior to systemic chemotherapy and/or prior to an extensive surgical resection. This would improve the selection of those who are most likely to benefit from an aggressive multimodal approach. However, evaluation of this indication for FDG$\mathrm{PET} / \mathrm{CT}$ in larger cohorts is warranted (28).

\section{Acknowledgements}

The authors wish to thank prof. Simon Horenblas for carefully reading the manuscript.

\section{Footnote}

Conflicts of Interest: The authors have no conflicts of interest to declare.

\section{References}

1. Kirrander P, Sherif A, Friedrich B, et al. Swedish National
Penile Cancer Register: incidence, tumour characteristics, management and survival. BJU Int 2016;117:287-92.

2. Barnholtz-Sloan JS, Maldonado JL, Pow-Sang J, et al. Incidence trends in primary malignant penile cancer. Urol Oncol 2007;25:361-7.

3. Arya M, Li R, Pegler K, et al. Long-term trends in incidence, survival and mortality of primary penile cancer in England. Cancer Causes Control 2013;24:2169-76.

4. Fletcher JW, Djulbegovic B, Soares HP, et al. Recommendations on the use of 18F-FDG PET in oncology. J Nucl Med 2008;49:480-508.

5. Lardinois D, Weder W, Hany TF, et al. Staging of nonsmall-cell lung cancer with integrated positron-emission tomography and computed tomography. N Engl J Med 2003;348:2500-7.

6. Antoch G, Saoudi N, Kuehl H, et al. Accuracy of wholebody dual-modality fluorine-18-2-fluoro-2-deoxy-Dglucose positron emission tomography and computed tomography (FDG-PET/CT) for tumor staging in solid tumors: comparison with CT and PET. J Clin Oncol 2004:22:4357-68.

7. Ng SH, Yen TC, Chang JT, et al. Prospective study of [18F]fluorodeoxyglucose positron emission tomography and computed tomography and magnetic resonance imaging in oral cavity squamous cell carcinoma with palpably negative neck. J Clin Oncol 2006;24:4371-6.

8. Scher B, Seitz M, Reiser M, et al. 18F-FDG PET/CT for staging of penile cancer. J Nucl Med 2005;46:1460-5.

9. Lont AP, Besnard AP, Gallee MP, et al. A comparison of physical examination and imaging in determining the extent of primary penile carcinoma. BJU Int 2003;91:493-5.

10. Kauhanen SP, Komar G, Seppänen MP, et al. A prospective diagnostic accuracy study of 18F-fluorodeoxyglucose positron emission tomography/computed tomography, multidetector row computed tomography, and magnetic resonance imaging in primary diagnosis and staging of pancreatic cancer. Ann Surg 2009;250:957-63.

11. Schöder H, Carlson DL, Kraus DH, et al. 18F-FDG PET/CT for detecting nodal metastases in patients with oral cancer staged N0 by clinical examination and CT/ MRI. J Nucl Med 2006;47:755-62.

12. Darling GE, Maziak DE, Inculet RI, et al. Positron emission tomography-computed tomography compared with invasive mediastinal staging in non-small cell lung cancer: results of mediastinal staging in the early lung positron emission tomography trial. J Thorac Oncol 2011;6:1367-72.

13. Leijte JA, Graafland NM, Valdés Olmos RA, et al. 
Prospective evaluation of hybrid 18F-fluorodeoxyglucose positron emission tomography/computed tomography in staging clinically node-negative patients with penile carcinoma. BJU Int 2009;104:640-4.

14. Souillac I, Rigaud J, Ansquer C, et al. Prospective evaluation of (18)F-fluorodeoxyglucose positron emission tomography-computerized tomography to assess inguinal lymph node status in invasive squamous cell carcinoma of the penis. J Urol 2012;187:493-7.

15. Sadeghi R, Gholami H, Zakavi SR, et al. Accuracy of 18F-FDG PET/CT for diagnosing inguinal lymph node involvement in penile squamous cell carcinoma: systematic review and meta-analysis of the literature. Clin Nucl Med 2012;37:436-41.

16. Clark PE, Spiess PE, Agarwal N, et al. Penile cancer: Clinical Practice Guidelines in Oncology. J Natl Compr Canc Netw 2013;11:594-615.

17. Graafland NM, Leijte JA, Valdés Olmos RA, et al. Scanning with 18 F-FDG-PET/CT for detection of pelvic nodal involvement in inguinal node-positive penile carcinoma. Eur Urol 2009;56:339-45.

18. Zhang S, Li W, Liang F. Clinical value of fluorine-18 2-fluoro-2-deoxy-D-glucose positron emission tomography/computed tomography in penile cancer. Oncotarget 2016;7:48600-6.

19. Uttam M, Pravin N, Anish B, et al. Is [F-18]fluorodeoxyglucose FDG-PET/CT better than ct alone for the preoperative lymph node staging of muscle invasive bladder cancer? Int Braz J Urol 2016;42:234-41.

20. Kitajima K, Murakami K, Yamasaki E, et al. Accuracy of 18F-FDG PET/CT in detecting pelvic and paraaortic lymph node metastasis in patients with endometrial cancer. AJR Am J Roentgenol 2008;190:1652-8.

Cite this article as: Ottenhof SR, Vegt E. The role of PET/ CT imaging in penile cancer. Transl Androl Urol 2017;6(5):833838. doi: $10.21037 /$ tau.2017.04.36
21. Atri M, Zhang Z, Dehdashti F, et al. Utility of PETCT to evaluate retroperitoneal lymph node metastasis in advanced cervical cancer: Results of ACRIN6671/ GOG0233 trial. Gynecol Oncol 2016;142:413-9.

22. Lin G, Chen CY, Liu FY, et al. Computed tomography, magnetic resonance imaging and FDG positron emission tomography in the management of vulvar malignancies. Eur Radiol 2015;25:1267-78.

23. Banzo J, Ubieto MA, Andrés A, et al. The contribution of ${ }^{18} \mathrm{~F}-\mathrm{FDG} \mathrm{PET} / \mathrm{CT}$ in a patient with cutaneous metastases of squamous cell carcinoma of the penis. Rev Esp Med Nucl Imagen Mol 2014;33:293-5.

24. Kaya ZR, Sager S, Halac M, et al. Disseminated metastatic penile squamous cell carcinoma detected by fluorodeoxyglucose PET/computerized tomography. Indian J Nucl Med 2012;27:189-91.

25. Sharma P. Late metastatic recurrence of penile carcinoma after 10 years: Demonstration with (18) F-fluorodeoxyglucose positron emission tomography/ computed tomography. Indian J Nucl Med 2016;31:204-6.

26. Leijte JA, Valdés Olmos RA, Mens JW, et al. Use of positron emission tomography in spindle cell carcinoma of the penis. Urology 2006;68:1121.e17-9.

27. Jakobsen JK, Alslev L, Ipsen P, et al. DaPeCa-3: promising results of sentinel node biopsy combined with (18) F-fluorodeoxyglucose positron emission tomography/ computed tomography in clinically lymph node-negative patients with penile cancer - a National study from Denmark. BJU Int 2016;118:102-11.

28. Barski D, Georgas E, Gerullis H, et al. Metastatic penile carcinoma - an update on the current diagnosis and treatment options. Cent European J Urol 2014;67:126-32. 